\title{
A New Fuzzy MADM Approach and its Application to Project Selection Problem
}

\author{
Ali Pahlavani \\ Department of Industrial Engineering, Iran University of Science and Technology, Narmak \\ Tehran, 16844, Iran \\ E-mail: pahlavani@iust.ac.ir
}

Received: 17-09-2008

Accepted: 27-11-2009

\begin{abstract}
In this paper, a novel fuzzy MADM model with some specifications that make it distinguished from the available methods. Decision matrix is defined as a full fuzzy structure. The model only uses information on the alternatives i.e. does not require pre-assigned weight values for the attributes. The weights will be achieved through a matching mechanism between the obtainable alternatives' preferences and a fuzzy preference relation of the alternatives stated by decision makers. This is applied using a mathematical programming model. Since the resulted model is non-linear and hard to solve using the classic optimization methods, Simulated Annealing is proposed to find optimum or near optimum weights. Having the weights of attributes, the alternatives' ranking is determined using the statistical measures of their fuzzy values. The model is applied on the project selection problem to study its efficiency and applicability to MADM problems.
\end{abstract}

Keywords: Fuzzy MADM, attribute, preference, Simulated Annealing, project selection

\section{Introduction}

Multi-criteria decision making can be characterized as making preferred decisions through evaluation, prioritization or selection of alternatives in the presence of multiple, usually conflicting criteria. Generally, multi-criteria decision making problems could be classified into two broad categories, namely MultiObjective Decision Making (MODM) and MultiAttribute Decision Making (MADM).

MADM refers to making decisions in a discrete decision space which is characterized by the explicit description of the set of alternatives and the attributes involved in the evaluation process. This type of problems arise in many real-world situations, e.g. the project selection or prioritization problem.

The most preferable state in a decision making situation, especially a MADM problem is when all aspects of the problem are known precisely. In many real world decision problems however, various kinds of uncertainty and vagueness exist which make the models more complex. In such situations, only for a small part of involved attributes, we can give exact assessments. For a main part of attributes, the evaluations are assessed as approximate values. Furthermore, for some attributes, we can only give linguistic assessments instead of exact assessments. ${ }^{1}$

The most important and well-known approach proposed to handle the uncertainty in various systems is fuzzy set theory first introduced by Zadeh. ${ }^{2}$ It has been increasingly applied in processing imprecise and uncertain information. Fuzzy set theory enables analyzers to describe the problem environment and its properties more close to reality and to build a more complex but still manageable model of the decision process.

Fuzzy set theory was first introduced into decision making by Bellman and $\mathrm{Zadeh}^{3}$ to handle the uncertainty involved in the problems. Since then, one of the interesting topics in this field has been dealing with imprecise discrete type decision problems through fuzzy based models. Fuzzy set theory in cooperation with 
MADM techniques offers good tools for handling such problems (see Refs. 4 and 5 for a survey on Fuzzy MADM methods).

This paper aims to develop a new fuzzy MADM model with some specifications which could be rarely found in the literature.

As we know, most MADM models need to define a set of values called weights reflecting relative importance of attributes. These weights are usually assumed to be pre-defined by decision makers. However it is a challenging step in problem solving process especially for the problems with a large number of attributes. To cope with reality and prevent from difficulties in determination of weights, our model proceeds without pre-assigned weights.

Nevertheless, the weights of attributes must be determined. In a basic classification, different approaches for calculating weights could be classified into two categories, objective and subjective. In objective approaches, the weights are calculated through the evaluation of decision matrix, representing the values of alternatives in attributes. The weights may change together with these values. An objective approach may comprise of multiple regression analysis, a modified weighted least square method and entropy. ${ }^{6}$

In the contrary, subjective methods for weight determination are based on decision makers' evaluation. They might express their preference on attributes or alternatives in specific style or may not indicate preference at all. Thus, different subjective methods could be classified into three categories ${ }^{7}$ : (1) the approaches without information, (2) information on attributes, and (3) information on alternatives.

In the third category, decision makers give their subjective perceptions in the form of preference information on alternatives.

As each decision makers comes from a different background and has his/her own ideas, attitudes, motivation and personality, different decision makers may provide their individual preference on alternatives in different formats. Authors in Ref. 8 explain different preference formats such as ordered vector, utility vector, selected subset, fuzzy selected subset, normal preference relations and fuzzy preference relation and utilize different approaches to convert all of the structures to a fuzzy preference relation.

Our model could be considered as a hybrid objective and subjective method based on preference information on alternatives. Here we suppose that the decision makers give this information in the format of fuzzy preference relation?

This is similar to the one developed by Fan et al. ${ }^{10}$ However, they assume that, the values of decision matrix are exactly defined. This is not the case in many real world problems such as our case study. Therefore, decision matrix in our model is defined as an array of fuzzy quantities. This leads to a full fuzzy approach for fuzzy MADM problems.

The attributes' weights are determined by minimizing the difference between decision makers' fuzzy preference relation and the preference of alternatives resulted from aggregating the values of decision matrix. The resulted non-linear mathematical programming model is solved by a meta-heuristic called Simulated Annealing.

The project selection problem is considered as a test bed for the developed model. The problem aims to select the appropriate project from a pool of projects or prioritize them with respect to some attributes with different aspects and directions.

The rest of paper is organized as follows. Section 2 gives the fundamental definitions of fuzzy theory. Section 3 describes the model in details. Section 4 takes a brief insight on the literature related to the case problem. In section 5, a practical example is given to demonstrate the applicability of the model. Section 6 presents some analytical experiments on the sensitivity of the model to change in inputs. Finally section 7 concludes the paper and proposes some issues for future research.

\section{Fuzzy Set Theory concepts}

In real-world modelling, we come face to face with different types of uncertainty. Fuzzy set theory is a powerful tool for dealing with such uncertainties.

Fuzzy set is a set with blur bounds. Unlike a conventional crisp set, which enforces either membership or non-membership of an object in a set, a fuzzy set allows grades of membership in the set. In this section, we give some essential definitions on fuzzy set theory. For extensive explanations of fuzzy sets readers are referred to the well-known references such as Zimmerman $^{11}$ and Klir, Clair and Yuan ${ }^{12}$.

Definition 1. A fuzzy set $\tilde{A}$ is defined by a membership function $\mu_{\tilde{A}}(x)$ which assigns to each object $x$ in the 
universe of discourse $X, a$ value in closed interval $[0,1]$ representing its grade of membership.

Definition 2. The fuzzy set $\tilde{A}$ is called to be convex if and only if:

$$
\begin{gathered}
\forall x_{1}, x_{2} \in X: \exists \lambda \in[0,1]: \\
\mu_{\tilde{A}}\left(\lambda x_{1}+(1-\lambda) x_{2}\right) \geq \operatorname{Min}\left(\mu_{\tilde{A}}\left(x_{1}\right), \mu_{\tilde{A}}\left(x_{2}\right)\right)
\end{gathered}
$$

Definition 3. The fuzzy set $\tilde{A}$ is called to be normal if $\exists x_{i} \in X, \mu_{\tilde{A}}\left(x_{i}\right)=1$.

Definition 4. Fuzzy number is a convex and normal fuzzy subset from universe $X$.

A generalized fuzzy number $\tilde{A}$ denoted by $(a, b, c, d)$ could be described as a fuzzy subset of the real line $R$, whose membership function $\mu_{\tilde{A}}(x)$ satisfies the following conditions.

- $\mu_{\tilde{A}}(x)$ is a continuous mapping from $R$ to the closed interval $[0,1]$,

- $\mu_{\tilde{A}}(x)=0,-\infty<x \leq a$,

- $\mu_{\tilde{A}}(x)=L(x)$ is strictly increasing on $[a, b]$,

- $\mu_{\tilde{A}}(x)=1, b \leq x \leq c$,

- $\mu_{\tilde{A}}(x)=R(x)$ is strictly decreasing on $[c, d]$,

- $\mu_{\tilde{A}}(x)=0, d \leq x<\infty$,

Decision makers are allowed to choose a variety of shapes as membership function such as triangular, trapezoidal, bell curves and s-curves ${ }^{12}$ based on their preferences.

Definition 5. A triangular fuzzy number (TFN) is denoted as $\tilde{A}=(a, b, d)$ and its membership function is as follows:

$$
\mu_{\tilde{A}}(x)=\left\{\begin{array}{llr}
\frac{x-a}{b-a}, & \text { if } & a \leq x \leq b \\
\frac{d-x}{d-b}, & \text { if } & b \leq x \leq d \\
0 & \text { else }
\end{array}\right.
$$

Fig. 1 is an illustration of the membership function of a triangular fuzzy number.

Same as crisp numbers, sometimes we need to apply arithmetic operations on fuzzy numbers. For this purpose, it is better to represent fuzzy numbers by their $\alpha$-level cuts and then to apply such operations on them.

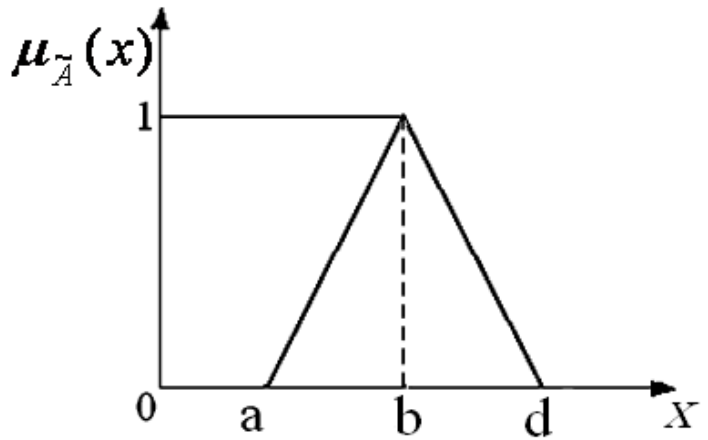

Fig. 1. Triangular fuzzy number.

Definition 6. For the fuzzy set $\tilde{A}$ its $\alpha$-level cut is defined as

$$
[\tilde{A}]_{\alpha}:\left\{x \mid \mu_{\tilde{A}}(x)>\alpha, 0 \leq \alpha \leq 1\right\}
$$

The fuzzy set $\tilde{A}$ can be expressed as

$$
\tilde{A}=\bigcup_{\alpha=0}^{1} \alpha \cdot[\tilde{A}]_{\alpha}
$$

where the symbol $\bigcup$ means maximum or disjunction operation in fuzzy logic.

From Def. 4, we know that the fuzzy numbers are convex fuzzy sets, so $\alpha$-level cuts are continual intervals. Using the definition of generalized fuzzy number, this interval will be:

$$
[\tilde{A}]_{\alpha}=\left[L^{-1}(\alpha), R^{-1}(\alpha)\right]
$$

Definition 7. If $\tilde{A}$ is a fuzzy number and all of its $\alpha$-level cuts are positive then $\tilde{A}$ is called a positive fuzzy number.

Regarding the above definitions, one can use the interval operations to do arithmetic operations on fuzzy numbers. With the assumption of $\left[a_{1}, b_{1}\right]$ and $\left[a_{2}, b_{2}\right]$ as two positive intervals, the arithmetic operations on them are defined as

$$
\begin{gathered}
{\left[a_{1}, b_{1}\right]+\left[a_{2}, b_{2}\right]=\left[a_{1}+a_{2}, b_{1}+b_{2}\right]} \\
{\left[a_{1}, b_{1}\right]-\left[a_{2}, b_{2}\right]=\left[a_{1}-b_{2}, b_{1}-a_{2}\right]} \\
{\left[a_{1}, b_{1}\right] \cdot\left[a_{2}, b_{2}\right]=}
\end{gathered}
$$

$\left[\min \left(a_{1} \cdot a_{2}, a_{1} \cdot b_{2}, b_{1} \cdot a_{2}, b_{1} b_{2}\right), \max \left(a_{1} \cdot a_{2}, a_{1} \cdot b_{2}, b_{1} \cdot a_{2}, b_{1} b_{2}\right)\right]$

$$
\left[a_{1}, b_{1}\right] /\left[a_{2}, b_{2}\right]=
$$




$$
\left[\min \left(\frac{a_{1}}{a_{2}}, \frac{a_{1}}{b_{2}}, \frac{b_{1}}{a_{2}}, \frac{b_{1}}{b_{2}}\right), \max \left(\frac{a_{1}}{a_{2}}, \frac{a_{1}}{b_{2}}, \frac{b_{1}}{a_{2}}, \frac{b_{1}}{b_{2}}\right)\right]
$$

Definition 8. If $\tilde{A}$ is a fuzzy number and $L^{-1}(\alpha)>0$ and $R^{-1}(\alpha) \leq 1$ for $\alpha \in[0,1]$, then $\tilde{A}$ is called a normalized positive fuzzy number.

Definition 9. $\tilde{D}$ is called a fuzzy matrix if at least one entry of it is a fuzzy number.

Definition 10. For a certain or crisp quantity as $k$ there is a corresponding triangular fuzzy number $\tilde{A}=(a, b, d)$ such that $a=b=d=k$.

Definition 11. For an approximate quantity such as "approximately $k$ " there is a corresponding triangular fuzzy number $\tilde{A}=(a, b, d)$ such that;

$$
\begin{gathered}
b=k \\
a=b .(1-(1-c r) .(1+s p)) \\
d=b .(1+(1-c r) .(1-s p))
\end{gathered}
$$

where $c r$ shows the confidence degree of decision maker about his/her approximation and $s p$ is the difference of right and left hand part of the membership function:

$$
s p=P_{L}-P_{R}
$$

where $P_{L}$ and $P_{R}\left(P_{L}+P_{R}=1\right)$ are respectively decision maker' idea about the possibility of being less or greater than $k$.

Definition 12. A linguistic variable is a variable whose values are linguistic terms.

Transformation of the fuzzy linguistic terms into triangular fuzzy numbers will be according to the rules described in Ref. 13 as depicted in Table 1.

Table 1. The relationship between linguistic terms and fuzzy numbers.

\begin{tabular}{cc}
\hline Fuzzy linguistic terms & Fuzzy numbers \\
\hline Very low (VL) & $(0,0,0.1)$ \\
Low (L) & $(0,0.1,0.3)$ \\
Medium low (ML) & $(0.1,0.3,0.5)$ \\
Medium (M) & $(0.3,0.5,0.7)$ \\
Medium high (MH) & $(0.5,0.7,0.9)$ \\
High (H) & $(0.7,0.9,1.0)$ \\
Very high (VH) & $(0.9,1.0,1.0)$ \\
\hline
\end{tabular}

\section{Fuzzy MADM model}

In a typical Fuzzy MADM problem, there are $m$ feasible alternatives $A_{1}, A_{2}, \ldots, A_{\mathrm{m}}$ which must be examined with respect to $n$ attributes, $C_{1}, C_{2}, \ldots, C_{\mathrm{n}}$. The weights of attributes are denoted by $w_{1}, w_{2}, \ldots, w_{\mathrm{n}}$.

The main requirement in each fuzzy MADM problem is to construct a decision matrix, $\tilde{D}$;

$\tilde{D}=\left[\begin{array}{cccc}\tilde{y}_{11} & \tilde{y}_{12} & \ldots & \tilde{y}_{1 n} \\ \tilde{y}_{21} & \tilde{y}_{22} & \ldots & \tilde{y}_{2 n} \\ \ldots & \ldots & \ldots & \ldots \\ \tilde{y}_{m 1} & \tilde{y}_{m 1} & \ldots & \tilde{y}_{m n}\end{array}\right]$

where $\tilde{y}_{i j}$ represents the assessment of the alternative $A_{\mathrm{i}}$ under the attribute $C_{\mathrm{j}}$. It can be a crisp number, approximate number, interval or linguistic words.

The values of non-fuzzy attributes will be converted to fuzzy numbers, through Defs. 10, 11 and 12.

To ensure compatibility between the evaluation values of different attributes, they must be converted into a compatible scale (dimensionless scale). This is achieved by normalizing the elements of decision matrix. The linear scale transformation can be used to normalize the various attribute scales.

First note that each attribute has either positive or negative direction. In the other words, there are two contradictory categories which would be called benefit $(B)$ and cost $(C)$. For a benefit attribute, much of it is preferable, but for a cost attribute, less of it is preferable.

If $\tilde{y}_{i j}=\left(y_{i j 1}, y_{i j 2}, y_{i j 3}\right)$ represents the TFN evaluation of the alternative $A_{i}$ under the attribute $C_{j}$, then the converted scale, $\tilde{x}_{i j}=\left(x_{i j 1}, x_{i j 2}, x_{i j 3}\right)$ can be calculated by:

$$
\begin{aligned}
& \tilde{x}_{i j}=\left(\frac{y_{i j 1}}{y_{j}^{*}}, \frac{y_{i j 2}}{y_{j}^{*}}, \frac{y_{i j 3}}{y_{j}^{*}}\right) \quad j \in B \\
& \tilde{x}_{i j}=\left(\frac{y_{j}^{-}}{y_{i j 3}}, \frac{y_{j}^{-}}{y_{i j 2}}, \frac{y_{j}^{-}}{y_{i j 1}}\right) \quad j \in C
\end{aligned}
$$

where $y_{j}^{*}=\max _{i} y_{i j 3}$ and $y_{j}^{-}=\min _{i} y_{i j 1}$.

Having the normalized fuzzy decision matrix, the overall evaluations of the alternatives could be obtained using Fuzzy Simple Additive Weighting method as Eq. (16):

$$
\tilde{d}_{i}=\sum_{j=1}^{n} \tilde{x}_{i j} \otimes w_{j}, i=1,2, \ldots, m
$$

\subsection{Fuzzy preference relation}

In this part we transform the overall values of the alternatives into a fuzzy preference relation. A fuzzy 
preference relation is a binary fuzzy relation $P$ in $A$, where $P$ is a mapping $A \times A \rightarrow[0,1]$ and $p_{i k}$ denotes the preference degree of alternative $A_{i}$ over $A_{k}$. In the matrix form, $P$ is reciprocal in which $p_{i k}+p_{k i}=1$ and $p_{i i}$ is defined as 0.5 .

To extract the fuzzy preference relation, we use the membership function of $\tilde{d}_{i}-\tilde{d}_{k}$ to indicate the preferability of alternative $A_{i}$ over $A_{k}$, and then assess $\tilde{d}_{i}-\tilde{d}_{k}$ whether it is smaller or larger than zero.

The difference $\tilde{d}_{i}-\tilde{d}_{k}$ could be easily calculated using $\alpha$-level cuts and applying Eq. (7) as follows:

$$
\begin{gathered}
\tilde{V}_{i k}=\tilde{d}_{i}-\tilde{d}_{k} \\
{\left[\tilde{V}_{i k}\right]_{\alpha}=\left[L_{i k}^{-1}(\alpha), R_{i k}^{-1}(\alpha)\right]}
\end{gathered}
$$

If $L_{i k}^{-1}(\alpha)>0$ for all $\alpha \in[0,1]$ then alternative $A_{i}$ absolutely preferred to alternative $A_{j}$ i.e. $\bar{p}_{i k}=1$. If $R_{i k}^{-1}(\alpha)<0$ for all $\alpha \in[0,1]$ then alternative $A_{k}$ absolutely preferred to alternative $A_{i}$ i.e. $\bar{p}_{i k}=0$. If $L_{i k}^{-1}(\alpha)>0$ for some values of $\alpha$ and $R_{i k}^{-1}(\alpha)<0$ for some others, we have ${ }^{14}$ :

$$
\begin{aligned}
& I_{1}=\int_{x>0} \mu_{\tilde{V}_{i k}}(x) d x \\
& I_{2}=\int_{x<0} \mu_{\tilde{V}_{i k}}(x) d x \\
& \bar{p}_{i k}=I_{1} /\left(I_{1}+I_{2}\right)
\end{aligned}
$$

In Eqs. (19)-(21), $I_{l}$ is the area below the negative part of the membership function of $\tilde{V}_{i k}$ and $I_{2}$ is the area below the positive part of the membership function of $\tilde{V}_{i k}$. This is illustrated in Fig. 2.

\subsection{Determination of the weights}

Since the weights of the attributes are not defined, Eq. (12) could not be applied over the alternatives. In order to calculate the weights of attributes, the preference relation derived from the overall evaluation values of alternatives (denoted by $\bar{P}$ ) will be compared with the preference relation of alternatives stated by decision makers. It is assumed that their subjective preference information on the alternatives is also in the form of fuzzy preference relation denoted by $P$.

The idea is based on minimizing the difference between the two preference relations. The overall difference between two preference relations is given by:

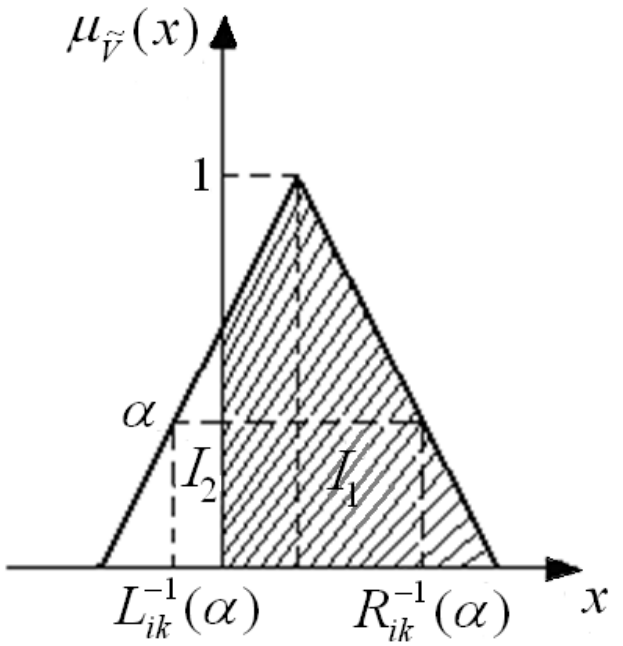

Fig. 2. Subtraction of two fuzzy numbers.

$$
O D=\sum_{j=1}^{m} \sum_{k=1}^{m}\left|\bar{p}_{i k}-p_{i k}\right|
$$

Since $\bar{p}_{i k}, i, k=1,2, \ldots, m$ are functions of the weights $w_{j}, j=1,2, \ldots, n, O D$ is also a function of them. Now we must minimize $O D$ by assessing the weights. So we have the following optimization model:

$$
\begin{aligned}
& \text { Minimize } O D(w)=\sum_{j=1}^{m} \sum_{k=1}^{m}\left|\bar{p}_{i k}-p_{i k}\right| \\
& \text { s.t. } \\
& \qquad \sum_{j=1}^{n} w_{j}=1 \\
& w_{j} \geq 0, j=1,2, \ldots, n
\end{aligned}
$$

If Eq. (16) is substituted by $w_{j}^{*}(j=1, \ldots, n)$, the optimum values of the weights, we can obtain the overall values of the alternatives.

Since the result of Eq. (16) is a fuzzy number, to obtain the final ranking of the alternatives and consequently the best alternative we apply the following procedure based on statistical concepts of fuzzy numbers.

For the fuzzy numbers $\tilde{d}_{i}=\left(d_{i 1}, d_{i 2}, d_{i 3}\right), i=1,2, \ldots, m$ resulted from Eq. (16), their mean value, standard deviation and coefficient of variation are calculated using the following equations ${ }^{15}$.

$$
\mu\left(\tilde{d}_{i}\right)=\frac{1}{4}\left(d_{i 1}+2 d_{i 2}+d_{i 3}\right)
$$




$$
\begin{gathered}
\sigma\left(\tilde{d}_{i}\right)=\frac{1}{80}\left(3 d_{i 1}^{2}+4 d_{i 2}^{2}+3 d_{i 3}^{2}-\ldots\right. \\
\left.\ldots 4 d_{i 1} \cdot d_{i 2}-4 d_{i 2} \cdot d_{i 3}-2 d_{i 1} \cdot d_{i 3}\right) \\
C V\left(\tilde{d}_{i}\right)=\frac{\sigma\left(\tilde{d}_{i}\right)}{\mu\left(\tilde{d}_{i}\right)} \quad \mu \neq 0, \sigma>0
\end{gathered}
$$

The ranking of alternatives will be according to the ascending order of their coefficient of variations. Therefore, the best alternative will be the one with the smallest coefficient of variation.

Although the model's steps are straightforward, program (P1) is non-linear and hard to solve because the objective function is a non-linear function of the weights. Therefore an approximation algorithm such as a meta-heuristic must be applied especially for large scale problems (the decision problems with a large number of attributes). The algorithm used is a very popular local search algorithm called Simulated Annealing explained in the following section.

\section{Simulated Annealing}

Simulated annealing (SA) first introduced by Kirkpatrick et al. ${ }^{16}$, finds its inspiration from the physical annealing process studied in statistical mechanics. SA repeats an iterative repairing procedure which looks for better solutions while offering the possibility of accepting worse solutions in a controlled manner. The second feature allows SA to escape from local optima.

In this paper we have modified SA to get a better efficiency in applying the algorithm. Figure 3 demonstrates a pseudo-code of the utilized algorithm.

The algorithm will be given an instance problem as the input. The instance could be denoted with the four parameters, $A$ as the set of alternatives, $C$ as the set of attributes, $\tilde{D}$ as the decision matrix and $P$ as the fuzzy preference relation matrix. The routine InitSol(.) gives an initial solution $\left(w_{0}\right)$ in which the weight of each attribute equals to $1 / n$. The best and current solutions of the algorithm are denoted respectively by $w^{*}$ and $w$.

After determination of initial temperature, a trial of predefined number of iterations (IterCount) is executed in an inner loop. Here a neighbor of a given solution $w$ is obtained by choosing randomly two attributes and subtracting a value, $\varepsilon$ from the weight of one attribute and adding it to the weight of another attribute.

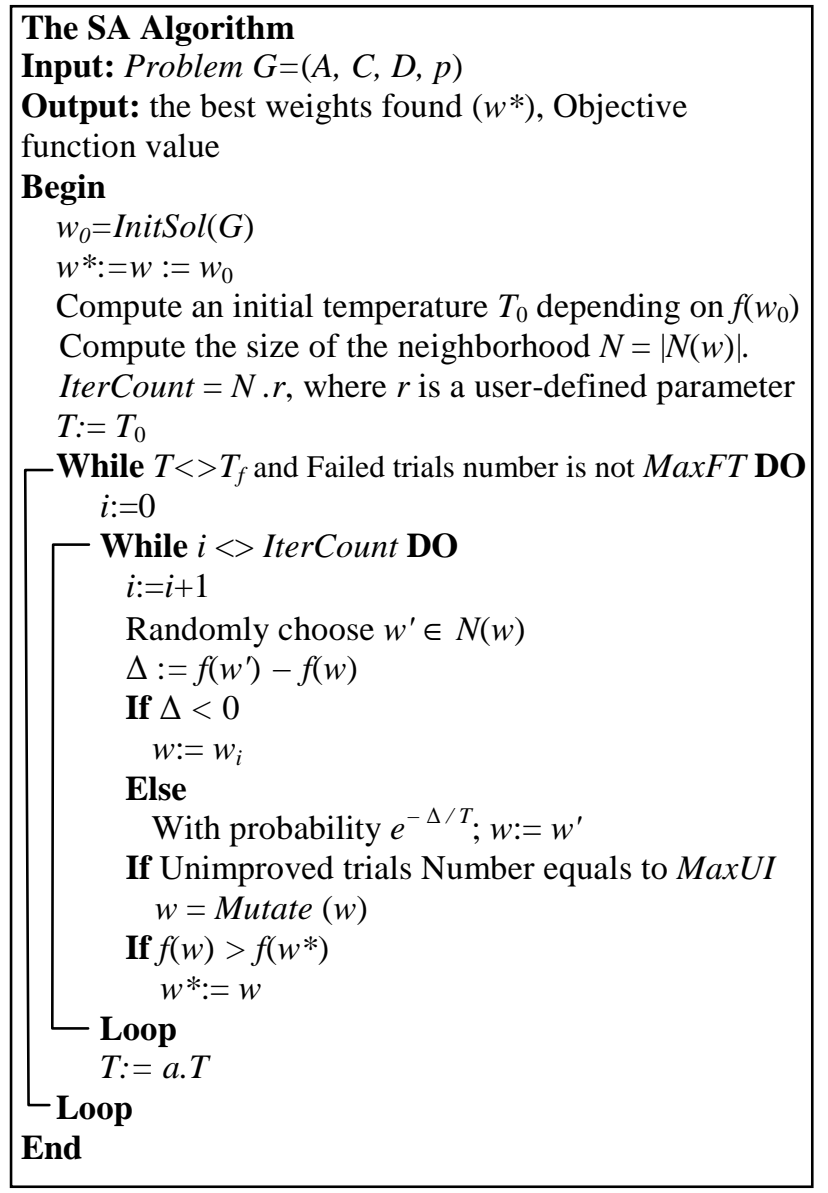

Fig. 3. A pseudo code of SA algorithm.

The current solution will be replaced by the generated neighbor according to SA mechanism.

More precisely, at each iteration, a neighbor $w^{\prime} \in N(w)$ of the current solution $w$ is generated randomly and a decision is then taken to decide whether $w^{\prime}$ will replace $w$. If $w^{\prime}$ is better than $w$ i.e. $\Delta=f\left(w^{\prime}\right)-f(w) \leq 0$ for minimization, we move from $w$ to $w^{\prime}$. Otherwise, we move to $w^{\prime}$ with the probability $e^{-\Delta / T}$. This probability depends on two factors: 1) the degree of the degradation $\Delta$ (smaller the degradation, greater the accepting probability), and 2) a control parameter $T$ called temperature.

The temperature is controlled by a cooling schedule specifying how the temperature should be progressively decreased. The cooling schedule function is given by $T_{k}=a . T_{k-1}$, in which $k$ is the current trial index. In this function, $a \in(0,1)$ is the temperature decreasing rate. Note that as $T$ decreases, the probability to accept worse 
solutions decreases. The algorithm is continued from the outer loop after updating the temperature.

To guide efficiently the algorithm to escape from local optima, a process similar to the mutation in Genetic Algorithm is inserted into the algorithm. It comes to course, if a predefined number of iterations stay unimproved during a trial. To mutate a solution, two attributes with the maximum and minimum weights are selected and their weight values are exchanged.

The algorithm stops when a pre-defined final temperature is reached. Because of computational considerations, another stop condition is applied on the algorithm. If the number of failed trials exceeds a predefined number (MaxFT), the algorithm terminates with the current best solution.

The framework of the model and its details were presented in this section. In the next section, emphasis will be on our case study and presentation of a brief survey on it.

\section{Project Selection Problem}

Project selection is the process of evaluating individual projects or a group of projects, and then choosing to implement some of them so that the objectives of the parent organization will be achieved. ${ }^{17}$

Project selection is a challenging problem faced by managers that deal with resource management and involves multiple attributes measuring rewards, relevance to the organization's mission and objectives, strategic leverage potential, probability of technical and commercial success, etc. ${ }^{18}$

Selecting the right project for future investment is a crucial decision for the long-term survival of a company. There is a large literature dedicated to the project selection problem and numerous techniques have been proposed in recent years for this problem.

Meredith $^{19}$ thoroughly describes the strategic intent of the project, factors for project selection, and various project selection models.

Qualitative and quantitative (numeric) are the two main categories of project selection methods. Organizations may use one of them or a combination of them.

A mostly-used approach in organizations is profitability analyzing for which financial methods as a subset of quantitative models can be used. The four common financial methods are ${ }^{20}$ : (1) Net Present Value,
(2) Rate of Return, (3) Benefit-Cost analysis, and (4) Pay Back Period.

Net present value (NPV) is a standard method for the financial appraisal of long-term projects. It is the difference between the sum of the discounted cash flows which are expected from the investment and the amount which is initially invested on the project.

Rate of return (ROR) is the percentage gain or loss of an investment over a period of time e.g. a year. A positive value for ROR corresponds to capital growth, a negative value corresponds to capital decay, and a value of $0 \%$ corresponds to no change.

Benefit-Cost Ratio (BCR) is an indicator, used in the formal discipline of cost-benefit analysis that attempts to summarize the overall value for money of a project. BCR is the ratio of the benefits of a project, expressed in monetary terms, relative to its costs, also expressed in monetary terms. All benefits and costs should be expressed in discounted present values.

Payback period (PP) refers to the period of time required for the return on an investment to repay the sum of the initial investment.

The main drawback of the above profitability models is their focus on a single decision criterion. The decision-making process for an investment selection must take into consideration both financial and nonfinancial effects and also both quantitative and qualitative effects. $^{18}$

A qualitative criterion which has an outstanding impact on the project financial success is project risk. Risk is a measure of the probability and consequence of not achieving a defined project goal. ${ }^{21}$ In each project whether small or large, complex or simple, risks are inevitable.

The requirement for considering multiple criteria in project evaluation process has enforced attempts to develop models that use multiple criteria to evaluate projects. Such models vary widely in their complexity and information requirements.

Analytic Hierarchy Process ${ }^{22,23,24}$ and TOPSIS ${ }^{25,26}$ are the two most important methods applied on this problem.

Definitely not all aspects of the evaluation easily measured because of their variety in quantitative and qualitative attitude.

Those with a financial viewpoint have a predictive context, so because of dynamic environment and 
unavailability of data or uncertainty become a matter of unreliability in estimation.

Some measures such as risk could be estimated only qualitatively which will force opinions and judgments. In fact, decision makers could not be able to provide a quantitative evaluation of the effect and of the implication of a project, but only a qualitative one.

So, tendency to apply fuzzy logic theory in this context is inevitable and we can enumerate some attempts. A fuzzy multi-objective programming using the fuzzy spatial algorithm for the problem of transportation investment project selection is presented in Ref. 27. A system for the project selection using fuzzy logic and based on uncertainty reduction is developed in Ref. 28.

Avineri et al. $^{29}$ presents a methodology for the selection and ranking of transportation projects using fuzzy set theory. Fuzzy ranking method is also proposed for evaluating and ranking manufacturing system investments ${ }^{30}$.

$\mathrm{Ng}$ et al. $^{31}$ has been to establish the fuzzy membership function of procurement selection criteria through an empirical study conducted in Australia.

Fuzzy analytical hierarchy process proposed to evaluate project risk in the selection level. ${ }^{32} \mathrm{~A}$ constrained version of fuzzy analytical hierarchy process is also applied in the field of project selection ${ }^{18}$.

Ramadan $^{33}$ developed a fuzzy MADM approach for R\&D project selection. It seems that the model is an extension of TOPSIS model in fuzzy environment. The author assumes that the weights of attributes are predefined.

Carlsson et al. ${ }^{34}$ developed a methodology for valuing options on $R \& D$ projects when future cash flows are estimated by trapezoidal fuzzy numbers.

Çekyay et al. ${ }^{35}$ introduced a new method that integrates Fuzzy Analytic Network Process (FANP) with Zero-one Goal Programming to solve Information System project selection problem. Goal Programming model selects the most appropriate project by considering both the weights of criteria found from Fuzzy ANP and constraints on resources.

Mahmoodzadeh et al. $^{36}$ proposed a method for project selection problem using fuzzy AHP and TOPSIS techniques. The attributes mentioned in the model are the above-mentioned methods for comparing investment alternatives.

A comprehensive review about project selection approaches is presented in chapter 26 of a well-known decision making book. ${ }^{37}$

\section{Example}

In this section, the developed model is examined through an example which comes from a case study applied in a commercial organization in Iran. We suppose that a set of feasible projects is defined. The focus will be on financial based attributes.

Let us assume that the organization has established a committee to get help in deciding the preferred project among four different projects $P_{1}, P_{2}, P_{3}$ and $P_{4}$ with respect to five attributes accounted for as methods for evaluating the profitability. The attributes are:

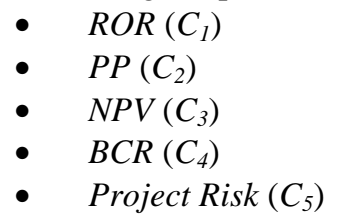

After relevant evaluations, the values of the projects on the attributes have submitted to the committee as Table 2 .

The evaluation values for the attributes $C_{1}, C_{2}, C_{3}$, and $C_{4}$ are approximation values in the form of $(k, r, s)$ as explained in Def. 11. The evaluations in attribute $C_{5}$ are given as linguistic terms. As we know, $C_{1}, C_{3}$ and $C_{4}$ are benefit type attributes and $C_{2}$ and $C_{5}$ are cost type attributes.

Table 2 presents a set of unrefined data and must be converted into an adaptable fuzzy format. Through Defs. 11 and 12, we can convert Table 2 into a unified fuzzy matrix (Table 3 ).

Using the procedure explained in Eqs. (14) and (15), the normalized decision matrix could be derived as shown in Table 4.

Now, the committee gives its preference on the projects in the form of fuzzy preference relation. Table 5 gives the overall fuzzy preference relation, derived from the individual fuzzy preference relations of the committee members. 
Table 2. The basic decision matrix

\begin{tabular}{cccccc}
\hline & $C_{1}$ & $C_{2}$ & $C_{3}$ & $C_{4}$ & $C_{5}$ \\
\hline$P_{1}$ & $(0.18,0.8,0)$ & $(36,0.5,-0.2)$ & $(86000,0.9,0)$ & $(2,0.8,0)$ & Medium high \\
$P_{2}$ & $(0.32,0.8,0)$ & $(35,0.5,-0.2)$ & $(91000,0.9,0)$ & $(1.7,0.8,0)$ & High \\
$P_{3}$ & $(0.22,0.8,0)$ & $(27,0.5,0)$ & $(82000,0.9,0)$ & $(1.5,0.8,0)$ & Medium \\
$P_{4}$ & $(0.2,0.7,0)$ & $(25,0.5,0)$ & $(74000,0.9,0)$ & $(1.2,0.8,0)$ & Medium low \\
\hline
\end{tabular}

Table 3. The fuzzy decision matrix

\begin{tabular}{cccccc}
\hline & $C_{l}$ & $C_{2}$ & $C_{3}$ & $C_{4}$ & $C_{5}$ \\
\hline$P_{1}$ & $(0.144,0.18,0.216)$ & $(21.6,36,57.6)$ & $(77400,86000,94600)$ & $(1.6,2,2.4)$ & $(0.5,0.7,0.9)$ \\
$P_{2}$ & $(0.256,0.32,0.384)$ & $(21,35,56)$ & $(81900,91000,100100)$ & $(1.36,1.7,2.04)$ & $(0.7,0.9,1)$ \\
$P_{3}$ & $(0.176,0.22,0.264)$ & $(13.5,27,40.5)$ & $(73800,82000,90200)$ & $(1.2,1.5,1.8)$ & $(0.3,0.5,0.7)$ \\
$P_{4}$ & $(0.14,0.2,0.26)$ & $(12.5,25,37.5)$ & $(66600,74000,81400)$ & $(0.96,1.2,1.44)$ & $(0.1,0.3,0.5)$ \\
\hline
\end{tabular}

Table 4. The normalized fuzzy decision matrix

\begin{tabular}{cccccc}
\hline & $C_{1}$ & $C_{2}$ & $C_{3}$ & $C_{4}$ & $C_{5}$ \\
\hline$P_{1}$ & $(0.375,0.469,0.563)$ & $(0.217,0.347,0.579)$ & $(0.773,0.859,0.945)$ & $(0.667,0.833,1.000)$ & $(0.111,0.143,0.200)$ \\
$P_{2}$ & $(0.667,0.833,1.000)$ & $(0.223,0.357,0.595)$ & $(0.818,0.909,1.000)$ & $(0.567,0.708,0.850)$ & $(0.100,0.111,0.143)$ \\
$P_{3}$ & $(0.458,0.573,0.688)$ & $(0.309,0.463,0.926)$ & $(0.737,0.819,0.901)$ & $(0.500,0.625,0.750)$ & $(0.143,0.200,0.333)$ \\
$P_{4}$ & $(0.365,0.521,0.677)$ & $(0.333,0.500,1.000)$ & $(0.665,0.739,0.813)$ & $(0.400,0.500,0.600)$ & $(0.200,0.333,1.000)$ \\
\hline
\end{tabular}

Table 5. The fuzzy preference relation

\begin{tabular}{ccccc}
\hline & $P_{1}$ & $P_{2}$ & $P_{3}$ & $P_{4}$ \\
\hline$P_{1}$ & 0.5 & 0.39 & 0.58 & 0.54 \\
$P_{2}$ & 0.61 & 0.5 & 0.62 & 0.57 \\
$P_{3}$ & 0.42 & 0.38 & 0.5 & 0.64 \\
$P_{4}$ & 0.46 & 0.43 & 0.36 & 0.5 \\
\hline
\end{tabular}

Having the normalized fuzzy decision matrix and the fuzzy preference relation, program $(P 1)$ could be applied. The model coded using MATLAB and the experiment with the algorithm is done with consideration of the following parameters:

- Initial temperature, $T_{0}=0.1$,

- Temperature decrease rate, $a=0.6$,

- Final temperature, $T_{\mathrm{f}}=0.00001$,

- Exchange value in trial $k, \varepsilon_{k}=T_{k}$,

- Iterations of each trial, IterCount $=30$,

- Maximum number of failed iterations, MaxUI=20,

- Maximum number of failed trials, MaxFT=5

After 580 iterations of algorithm running, the best value found for the objective value was 0.2169 . The trend of objective function value in different iterations is given in Fig. 4. The weight vector of the attributes is obtained as:

$$
w^{*}=(0.1668,0.1874,0.1742,0.2966,0.1751)
$$

Using fuzzy simple additive weighting method, the overall fuzzy values of the alternatives is as Table 6.

According to the ranking process of the overall fuzzy values based on Eqs. (22), (23) and (24), the resulted ranking is $P_{2}>P_{1}>P_{3}>P_{4}$ as shown in Table 6. Thus, project $P_{2}$ is selected due to proposed approach.

\section{Further Analysis}

This section considers some sensitivity analyses on the proposed model.

Analyses are carried out to show how the model is sensitive to change in the elements of decision matrix and also the preference relations. Table 7 exemplifies the remarkable changes in the final result by adjusting one element in the decision matrix while leaving the others unchanged.

Table 6. The ranking of alternatives

\begin{tabular}{cccccc}
\hline Project & Overall Fuzzy Value & Mean Value & Standard Deviation & Coefficient of Variation & Ranking \\
\hline$P_{1}$ & $(0.4551,0.5649,0.6986)$ & 0.57087 & 0.00149 & 0.00261 & $\mathbf{2}$ \\
$P_{2}$ & $(0.4812,0.5935,0.7295)$ & 0.59945 & 0.00155 & 0.00258 & $\mathbf{1}$ \\
$P_{3}$ & $(0.4360,0.5453,0.7259)$ & 0.56314 & 0.00216 & 0.00384 & $\mathbf{3}$ \\
$P_{4}$ & $(0.3927,0.5159,0.7949)$ & 0.55486 & 0.00435 & 0.00784 & $\mathbf{4}$ \\
\hline
\end{tabular}


Table 7. Sensitivity of the weights and ranking to change in decision matrix

\begin{tabular}{ccccc}
\hline Item & $\begin{array}{c}\text { Change } \\
\text { to }\end{array}$ & $\begin{array}{c}\text { Objective } \\
\text { Value }\end{array}$ & Weights & Ranking \\
\hline Value & 0.2 & 0.2178 & 0.1910 & $P_{2}$ \\
of $P_{1}$ & & & 0.2049 & $P_{1}$ \\
in $C_{1}$ & & & 0.1506 & $P_{3}$ \\
& & & 0.2847 & $P_{4}$ \\
\hline Value & 40 & 0.2524 & 0.1687 & \\
of $P_{2}$ & & & 0.2460 & $P_{2}$ \\
in $C_{2}$ & & & 0.2459 & $P_{1}$ \\
& & & 0.3618 & $P_{3}$ \\
& & & 0.1300 & $P_{4}$ \\
\hline Value & 100,000 & 0.2154 & 0.1931 & $P_{2}$ \\
of $P_{3}$ & & & 0.1201 & $P_{1}$ \\
in $C_{3}$ & & & 0.0408 & $P_{3}$ \\
& & & 0.3891 & $P_{4}$ \\
& & & 0.2569 & \\
\hline Value & 2 & 0.2266 & 0.0307 & $P_{2}$ \\
of $P_{4}$ & & & 0.1850 & $P_{1}$ \\
in $C_{4}$ & & & 0.5832 & $P_{3}$ \\
& & & 0.1175 & $P_{4}$ \\
\hline Value & Medium & 0.2234 & 0.0310 & $P_{2}$ \\
of $P_{2}$ & low & & 0.2976 & $P_{1}$ \\
in $C_{5}$ & & & 0.1595 & $P_{3}$ \\
& & & 0.3464 & $P_{4}$ \\
\hline
\end{tabular}

As it can be seen from Table 7, different weight values are determined for different decision matrices. The resulted ranking however, does not change for none of the changes. This outcome is because the ranking is strictly dependant on the original preference relation and the model determines the weights such that final ranking complies with the ranking obtained using the fuzzy preference relation. However, in the cases which the objective value is high i.e. the derived preference relation complies slightly with the original preference relation, any change in the ranking is possible.

Therefore, the ranking might change by applying some changes in the preference relation matrix. Two examples are shown in Table 8.

Table 8. Sensitivity of the weights and ranking to change in preference relation

\begin{tabular}{cccccc}
\hline Item & $\begin{array}{c}\text { Change } \\
\text { from }\end{array}$ & $\begin{array}{c}\text { Change } \\
\text { to }\end{array}$ & $\begin{array}{c}\text { Objective } \\
\text { Value }\end{array}$ & Weights & Ranking \\
\hline Preference & 0.39 & 0.61 & 0.3029 & 0.0032 & $P_{1}$ \\
of $P_{1}$ to $P_{2}$ & & & & 0.3067 & $P_{2}$ \\
& & & & 0.2421 & $P_{3}$ \\
& & & & 0.3632 & $P_{4}$ \\
\hline Preference & 0.62 & 0.38 & 0.3566 & 0.0469 & $P_{1}$ \\
of $P_{2}$ to $P_{3}$ & & & & 0.4250 & $P_{2}$ \\
& & & & 0.3203 & $P_{3}$ \\
& & & & 0.2076 & $P_{4}$ \\
\hline
\end{tabular}

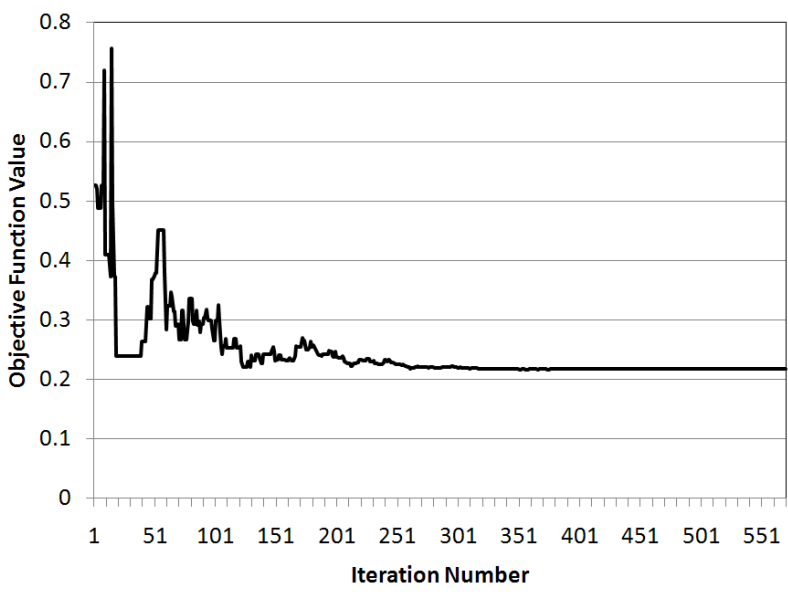

Fig. 4. The trend of objective function value in the experiment with SA.

\section{Conclusions and Future Research}

In this paper, we have considered a special class of fuzzy MADM problems is which the prior information is only the fuzzy preference relation of alternatives. So, the attributes' weights reflecting their relative importance are not pre-defined. The decision makers don't need to express their opinions about the attributes' importance to each other.

A new Fuzzy MADM model has been developed to derive the weight values of the attributes. The weights are obtained by comparing the decision makers' preference relation of alternatives with the preference relation derived from aggregating the values of the alternatives included in decision matrix. It is applied through a mathematical programming model.

Since the program is non-linear and hard to solve to optimality, we have proposed to solve the model using Simulated Annealing.

The model uses Fuzzy Simple Additive Weighting Method to get the overall values of alternatives as fuzzy numbers. Although, using other methods available in literature for aggregation is also possible.

Efficiency of the model has been analyzed through a case study in a commercial organization involved in an important decision - selecting the most preferred project from the profitability view point.

The developed model is capable of working with a broad spectrum of decision making problems. However, it is most applicable on the problems with a small number of alternatives and a large number of attributes. 
Unlike most MADM models that work with crisp decision matrices, our model applies a fuzzy decision matrix and does not need any defuzzification.

In the problems which the preference information on the attributes is also available, we can calculate the subjective weight values and combine them with the objective weights resulted from our model using aggregation operators such as arithmetical or geometric mean.

Another possibility for improving the developed model would be to consider weight values as fuzzy numbers same as decision matrix.

\section{Acknowledgements}

The author would like to thank two anonymous referees for their valuable comments which improved the contribution.

\section{References}

1. H. Liu and F. Kong, A new MADM algorithm based on fuzzy subjective and objective integrated weights, Int. J. Inf. Sys. Sci. 1 (3-4) (2005) 420-427.

2. L.A. Zadeh, Fuzzy sets, Inf. Control. 8 (1965) 338-353.

3. R.E. Bellman and L.A. Zadeh, Decision making in a fuzzy environment, Manag. Sci. 17 (4) (1970) B141B164.

4. S.J. Chen and C.L. Hwang, Fuzzy Multiple Attribute Decision Making: Methods and Applications, (SpringerVerlag, Berlin, 1992).

5. J. Figueria, S. Greco and M. Ehrgott, Multiple Criteria Decision Analysis: state of the art surveys, (Springer Science, USA, 2005).

6. R. Ginevičius and V. Podvezko, Objective and subjective approaches to determining the criterion weight in multicriteria models, Transp. Telecommun. 6 (1) (2005) 133-137.

7. C.L. Hwang and K. Yoon, Multiple Attribute Decision Making: Methods and Applications, (Springer-Verlag, Berlin, 1981).

8. J. Ma, Q. Zhang, D. Zhou and Z. P. Fan, A multiple person multiple attribute decision making method based on preference information and decision matrix, working paper, Working paper, Department of Information Systems, City University of Hong Kong, address: http://www.is.cityu.edu.hk/Research/WorkingPapers/pap er/0006.pdf, accessed: 2008/12/02.

9. S.A. Orlovski, Decision-making with a fuzzy preference relation, Fuzzy Sets. Syst. 1 (1978) 155-167.

10. Z. Fan, J. Ma and Q. Zhang, An approach to multiple attribute decision making based on fuzzy preference information on alternatives, Fuzzy Sets. Syst. 131 (1) (2002) 101-106.
11. H.J. Zimmerman, Fuzzy Set Theory and its Applications, 2nd edn. (Kluwer Academic Publishers, Boston, 1991).

12. G.J. Klir, U. St. Clair, and B. Yuan, Fuzzy Set Theory: Foundations and Applications (Prentice Hall, Englewood cliffs, NJ, 1997).

13. C. T. Chen, Extension of the TOPSIS for group decision making under fuzzy environment, Fuzzy Sets. Syst. 114 (1) (2000) 1-9.

14. C. T. Chen, A fuzzy approach to select the location of the distribution center, Fuzzy Sets. Syst. 118 (2001) 65-73.

15. M. R. Mehregan and H. Safari, Combination of fuzzy TOPSIS and fuzzy ranking for multi attribute decision making, in Proc. 8th Int. Conf. Artificial Intelligence and Soft Computing, eds. L. Rutkowski, R. Tadeusiewicz, L. A. Zadeh and J. Zurada (Springer-Verlag, Berlin, 2006), pp. $260-267$.

16. S. Kirkpatrick, CD. Gellatt, and MP. Vecchi, Optimization by simulated annealing, Sci. 220 (4598) (1993) 671-680.

17. H. Eilat, B. Golany and A. Shtub, R\&D project evaluation: An integrated DEA and balanced scorecard approach, Omega. 36 (5) (2008) 895-912.

18. M. Enea and T. Pizza, Project selection by constrained Fuzzy AHP, Fuzzy Optim. Decis. Mak. 3 (1) (2004) 3962.

19. J.R. Meredith and S.J. Mantle, Project Management: a managerial approach (Wiley, New York, 2003).

20. R. Burke, Project Management: Planning and Control Techniques, 4th edn. (Wiley, Hoboken, NJ, 2003).

21. N.D.P. Shull, "Project evaluation using fuzzy logic and risk analysis techniques" MSc Thesis, (University of Puerto Rico, 2006).

22. P. Ferrari, A method for choosing from among alternative transportation projects, Eur. J. Oper. Res. 150 (1) (2003) 194-203.

23. G.S. Kearns, A multi-objective, multi-criteria approach for evaluating IT investments: results from two case studies, Inf. Resour. Manag. J. 17 (1) (2004) 37-62.

24. I. M. Mahdi and K. Alreshaid, Decision support system for selecting the proper project delivery method using analytical hierarchy process, Int. J. Proj. Manag. 23 (7) (2005) 564-572.

25. P. K. Dey, Integrated project evaluation and selection using multiple-attribute decision-making technique, Int. J. Prod. Econ. 103 (1) (2006) 90-103.

26. Y. Chen, W.-d. Chen, J. Peng and J.-y. Wang, TOPSIS approach to project selection in six- $\sigma$ management, Ind. Eng. J. 8 (4) (2005) 90-92.

27. J.Y. Teng and G.H. Tzeng, Transportation investment project selection using fuzzy multiobjective programming, Fuzzy Sets. Syst. 96 (3) (1998) 259-280.

28. L. L. Machacha and P. Bhattacharya, A fuzzy-logicbased approach to project selection, IEEE Trans. Eng. Manag. 47 (1) (2000) 65-73.

29. E. Avineri, J. Prashker and A. Ceder, Transportation projects selection process using Fuzzy sets theory, Fuzzy Sets. Syst. 116 (1) (2000) 35-47. 
30. S. Coldrick, C.P. Lawson, P.C. Ivey, and C. Lockwood, A decision framework for R\&D project selection, in Proc. the IEEE Int. Engineering Management Conf. (United Kingdom, Cambridge, 2002), pp. 413-418.

31. S. T. Ng, D. T. Luu, S. E. Chen and K. C. Lam, Fuzzy membership functions of procurement selection criteria, Constr. Manag. Econ. 20 (3) (2002) 285-296.

32. F. Tüysüz and C. Kahraman, Project risk evaluation using a fuzzy analytic hierarchy process: an application to information technology projects, Int. J. Intell. Syst. 21 (6) (2004) $559-584$.

33. M. Z. Ramadan, A fuzzy model for R\&D project selection with multi-criteria decision making, in Proc. 2nd Int. Industrial Engineering Conf. (Kingdom of Saudi Arabia, Riyadh, 2004), pp. 1-11.

34. C. Carlsson, R. Fullér, M. Heikkilä and P. Majlender, A fuzzy approach to R\&D project portfolio selection, Int. J. Approx. Reason. 44 (2) (2007) 93-105.

35. B. Çekyay, Ç. A. Gümüssoy and T. Ertay, IS project selection based on FUZZY-ANP and ZOGP, in Proc. 35th Int. Conf. Computers and Industrial Engineering, (Turkey, Istanbul, 2005), pp. 475-482.

36. S. Mahmoodzadeh, J. Shahrabi, M. Pariazar, and M. S. Zaeri, Project election by using Fuzzy AHP and TOPSIS technique, Int. J. Humanit. Soc. Sci. 1 (3) (2007) 135140.

37. R. Venkata Rao, Decision Making in the Manufacturing Environment: Using Graph Theory and Fuzzy Multiple Attribute Decision Making Methods, (Springer, London, 2007). 